\title{
Reactive Market Power Analysis Using Must-Run Indices
}

\author{
Donghan Feng, Jin Zhong, Member, IEEE, and Deqiang Gan
}

\begin{abstract}
This paper investigates the issues of reactive power must-run capacity in power system operations, hence in electricity markets. A must-run index-based method is proposed in the paper to measure the market power held by reactive power suppliers. The Nordic 32-bus system and the IEEE 118-bus system are used to test the proposed method. The market power holders of reactive power found using the proposed method are in accord with that found in the realistic Nordic system operation and in the existing analysis of IEEE 118-bus system. The paper identifies through must-run indices possible conditions that could lead to market power in the case of applying a bid structure within a market framework. Furthermore, market structure drawbacks can cause the appearance of market power even in a topologically ideal system.
\end{abstract}

Index Terms-Electricity market, market power, must-run index, reactive power.

\section{INTRODUCTION}

$\mathbf{T}$ HE issues about reactive power pricing mechanisms have been discussed for a long time even before power system deregulation. Voltage control and reactive power support is considered as a transmission service provided by the vertically integrated power company. The system operator usually procures reactive power services through long-term contracts.

With the deregulation of power industry, real power, which had been centrally dispatched and sold to customers in the traditional power systems, become the first product to be auctioned and sold in electricity markets. The transmission services provided by the system operators for system stability and reliability are still provided centrally and not yet being traded in the markets. With the further development of electric energy market and electricity financial market, more and more services are, or will be traded in the ancillary service market, for example, regulation service is traded in the electricity balancing markets of Nordic countries, and reserves are traded in the ancillary service markets in some of the American electricity markets. The market mechanisms of reactive power service probably will be the next one being discussed in the ancillary service markets.

In the current restructured power industries, reactive power management and payment mechanisms vary for different mar-

Manuscript received December 27, 2006; revised December 28, 2007. This work was supported in part by the Research Grand Council of Hong Kong under Grant HKU7201/05E and in part by the HKU Seed Funding Program for Basic Research (project code: 200411159086). Paper no. TPWRS-00904-2006.

D. Feng and D. Gan are with the College of Electrical Engineering, Zhejiang University, Hangzhou, China (e-mail: seedplayer@163.com; deqiang.gan@ieee.org)

J. Zhong is with the Department of Electrical and Electronic Engineering, The University of Hong Kong, Hong Kong (e-mail: jzhong@eee.hku.hk).

Color versions of one or more of the figures in this paper are available online at http://ieeexplore.ieee.org.

Digital Object Identifier 10.1109/TPWRS.2008.919416 kets. In the U.S., reactive power provided by a generator is considered as an ancillary service and is compensated with a certain fixed payment [1]. In Australia, the reactive power ancillary service is compensated by availability component, enabling component, and compensation component [2].

With the development of ancillary service markets, there is a trend to procure reactive power in a competitive way. In the past several years, there were some discussions about the possibility of establishing a reactive power market. In [3], the reactive power market arrangement based on reactive power capacity payment and utilization payment was discussed and analyzed using a security constrained optimal power flow (OPF) model. A two-segment reactive power pricing framework was proposed in [4]. Mechanisms for reactive energy pricing and reactive power capacity pricing were presented in the paper. A competitive market framework for reactive power was proposed in [5]. In the framework, a reactive power bidding structure [6] was proposed and the reactive power provided by generators was settled at a uniform market price.

However, due to the localized characteristics of reactive power, the market power problem for reactive power is more serious than real power, which impedes the implementation of reactive power market. Reactive market power has been observed in [3], [5], and [7] that some reactive power suppliers can manipulate reactive power market prices.

In this paper, we will first develop must-run indices to analyze the market power problem for reactive power from both network and market aspects. The topology of an electric power system may result in inherent market power, which exists in the system, no matter it is a monopoly system or a competitive market. While, effective market regulations or a well-designed market structure can mitigate or eliminate the market power in the system, hence prevent market power holder from exercising market power; a not well-designed market may worsen the situation. Based on the proposed must-run indices, we will then study the impacts of market structure on reactive market power, as well as the levels of market power held by a generator and a generation company (GenCo).

In Section II, existing market power measurement tools are introduced. In Section III, the must-run-based indices are proposed for measuring reactive market power for individual generators and GenCos, respectively. In Section IV, the Nordic 32-bus system and the IEEE 118-bus system are used to test the proposed indices. The conclusions are drawn in Section V.

\section{MARKET POWER MEASUREMENT INDICES}

Market power of traditional industry structures are measured by some commonly used indices. The indices include 
Herfindahl-Hirschman Index (HHI), Entropy Coefficient (EC) and Lerner Index (LI), etc. [8], [9].

Both HHI and EC are calculated according to the market shares of suppliers. The indices are formulated as (1) and (2), respectively

$$
\begin{gathered}
H H I=10000 \sum_{i=1}^{N} s_{i}^{2} \\
E C=-\sum_{i=1}^{N} s_{i} \ln \left(s_{i}\right)
\end{gathered}
$$

where $s_{i}$ refers to the market share of participant $i, N$ refers to the number of participants in the market. For the highest concentration of a market, which is a monopoly structure, $H H I=$ 10000 . The higher concentration the market share, the higher the HHI. Opposite to HHI, a high EC represents a low market concentration, and $E C=0$ for monopoly structure.

Unlike HHI and EC, LI measures the degree of exercising market power by comparing market price with marginal cost. For a market that is not completely competitive, the market price is higher than marginal cost. The deviation of market price from marginal cost can represent the level of exercising market power. LI is formulated as

$$
L I=\frac{P-M C}{P}
$$

where $P$ is market price and $M C$ is marginal cost.

Originally, $\mathrm{HHI}$ and EC are designed to measure market concentrations, and LI is designed to measure demand restrictions on monopoly power. There are some limitations of applying HHI and LI directly in power systems. HHI considers only market concentration and it is hard to consider power system operation information. For LI, the demand elasticity is not very clear to each generator for LI calculation.

Must-run ratio (MRR) was proposed in [10] for measuring the locational market power in an electric energy market. In a power system, the must-run capacity (MRC) of a generator, say generator $A$, is calculated as the system generation shortage in the case that generator $A$ is out of service. In other words, to satisfy system demand, generator $A$ must generate at least a certain amount of must-run capacity. The must-run ratio of generator $A$ is defined as

$$
M R R=\frac{\text { Must Run Capacity of } A}{\text { Capacity of } A} .
$$

Must-run share indices were developed in [11] to evaluate the impacts of load variations, transmission constraints and failures on market power. Market power exists not only in real power systems, but also in reactive power systems. Recent years, some methods have been used for measuring the market power of reactive power. The HHI index was first introduced in [12] to measure the market share of a generator in reactive power supply. Another measurement of reactive power market shares was defined in [13]. The method considers the effects of the generators that reach reactive power limits. In these methods, market power of reactive power was measured mostly based on market shares and market concentration indices.
In Section III, we will propose must-run indices to measure the reactive market power from different aspects while considering reactive power capability and system voltages.

\section{Must-Run Indices FOR REACTIVE MARKET POWER MEASUREMENT}

To consider the reactive power must-run capacity of a system, two issues need to be addressed:

- voltage profile of the system;

- network structure and the locations of reactive power suppliers.

To determine whether a generator is reactive power must-run, a simple concept can be applied. Assume there is a scenario that the reactive power output of a generator is zero, if system voltages can still be maintained within voltage limits, this generator is not reactive power must-run (or, incapable of exercising market power). In some systems, or for certain operation conditions, some generators are compulsory to contribute certain amount of reactive power to maintain system voltages. We call these generators reactive power must-run generators, i.e., they hold reactive market power. The amount of the must-run capacity of a generator represents the level of market power the generator holds.

If a GenCo owns at least one reactive power must-run generator, we say that the GenCo has reactive market power. In power system operations, a GenCo without must-run generator could also hold market power by manipulating the reactive power output of its generators. We will analyze the must-run capacities of GenCos as well as individual generators. The must-run indices are proposed for generators and GenCos respectively in Sections III-A and III-B.

\section{A. Indices for Measuring Market Power for Individual Generators}

To estimate the amount of must-run capacity of a generator, we start from the initial reactive power scheme and the voltage profile obtained by the OPF solution. Assume in a normal operation condition, the voltage of bus $j$ is $V_{j}^{0}$, and the reactive power scheduled for the generator at bus $i$ is $Q_{i}^{0}$. In the case that generator $i$ has no reactive power output, if the voltage of bus $j$ can still be maintained within voltage limit $\left[V_{j}, \overline{V_{j}}\right]$ by reactive power supported from other generators and $\mathrm{VAr}$ devices, we say that generator $i$ has no market power on bus $j$. The generator is not "must-run" for maintaining the voltage of bus $j$. On the other hand, if generator $i$ is a must-run generator for supporting the voltage of bus $j$, we can evaluate the level of its market power on bus $j$ by calculating its reactive power must-run capacity. Here, we propose four steps to calculate the reactive must-run capacity of a generator.

First, the possible voltage drop on bus $j$ caused by generator $i$ withholding reactive power is calculated. The voltage drop on bus $j$ from the initial voltage $V_{j}^{0}$ is formulated in

$$
V_{i \rightarrow j}^{-}= \begin{cases}\left(Q_{i}^{0}-Q_{i}\right) \cdot \frac{\partial V_{j}}{\partial Q_{i}}, & \frac{\partial V_{j}}{\partial Q_{i}}>0 \\ \left(Q_{i}^{0}-\overline{\overline{Q_{i}}}\right) \cdot \frac{\partial V_{j}}{\partial Q_{i}}, & \frac{\partial V_{j}}{\partial Q_{i}}<0 \\ 0, & \frac{\partial V_{j}}{\partial Q_{i}}=0\end{cases}
$$


where $V_{i \rightarrow j}^{-}$is the voltage drop on bus $j$ due to generator $i$ 's reactive power reduction from initial value $Q_{i}^{0}$ to $\underline{Q_{i}}$, which is the reactive power lower limit of generator $i$. Here, the superscript "-" refers to a negative effect (voltage drop). $\partial V_{j} / \partial Q_{i}$ denotes the sensitivity of bus voltage to reactive power injection. For a positive sensitivity, the voltage on bus $j$ will drop if generator $i$ withhold its reactive power. For a negative sensitivity, the voltage on bus $j$ will drop when generator $i$ generate more reactive power. Equation (5) can be equivalently formulated as

$$
\begin{aligned}
V_{i \rightarrow j}^{-}=\left(Q_{i}^{0}-\underline{Q_{i}}\right) \cdot \max & \left(\frac{\partial V_{j}}{\partial Q_{i}}, 0\right) \\
& +\left(Q_{i}^{0}-\overline{Q_{i}}\right) \cdot \min \left(\frac{\partial V_{j}}{\partial Q_{i}}, 0\right) .
\end{aligned}
$$

Second, the voltage supports from other reactive power facilities are calculated. The voltage change on bus $j$ caused by the reactive power sources other than generator $i$ is formulated as $V_{-i \rightarrow j}^{+}$in (7). The superscript "+" refers to positive effect (or voltage support), and subscript " $-i \rightarrow j$ " refers to all reactive power facilities other than generator $i$ that support voltage on bus $j$

$$
\begin{aligned}
V_{-i \rightarrow j}^{+}= & \sum_{k \neq i, k \in G \bigcup C}\left(\overline{Q_{k}}-Q_{k}^{0}\right) \cdot \max \left(\frac{\partial V_{j}}{\partial Q_{k}}, 0\right) \\
& +\sum_{k \neq i, k \in G \bigcup C}\left(\underline{Q_{k}}-Q_{k}^{0}\right) \cdot \min \left(\frac{\partial V_{j}}{\partial Q_{k}}, 0\right)
\end{aligned}
$$

where $\overline{Q_{k}}$ and $Q_{k}$ represent the reactive power upper and lower limits for the $\mathrm{VAr}$ supplier on bus $k$, respectively. $G$ and $C$ denote, respectively, the index set of generators and the index set of reactive power devices, such as shunt capacitors and FACTS facilities.

Third, by summing up the negative and positive deviation effects on $V_{j}$ that formulated in (6) and (7), we can obtain the voltage deficit on bus $j$ in the case that generator $i$ manipulate its reactive power output. The voltage deficit $\Delta V_{i \rightarrow j}$ is given in

$$
\Delta V_{i \rightarrow j}=\max \left\{0,\left(V_{i \rightarrow j}^{-}-V_{-i \rightarrow j}^{+}\right)-\left(V_{j}^{0}-\underline{V_{j}}\right)\right\} .
$$

Item $\left(V_{i \rightarrow j}^{-}-V_{-i \rightarrow j}^{+}\right)$denotes the residual voltage drop on bus $j$ due to the reactive power withheld by generator $i$. $\left(V_{j}^{0}-\underline{V_{j}}\right)$ denotes the voltage margin between the initial voltage and the lower limit. If the residual voltage drop $\left(V_{i \rightarrow j}^{-}-V_{-i \rightarrow j}^{+}\right)$is greater than the voltage margin $\left(V_{j}^{0}-\underline{V_{j}}\right)$, or $\left(V_{i \rightarrow j}^{-}-V_{-i \rightarrow j}^{+}\right)-\left(V_{j}^{0}-\underline{V_{j}}\right)$ is positive, the voltage on bus $j$ cannot be maintained (by readjusting other facilities' reactive power output) within voltage limits when generator $i$ withholds its reactive power output. In this situation, there is a voltage deficit $\Delta V_{i \rightarrow j}$ to reach $V_{j}$. If the residual voltage drop is less than voltage margin, or $\left(V_{i \rightarrow j}^{-}-V_{-i \rightarrow j}^{+}\right)-\left(V_{j}^{0}-\underline{V_{j}}\right)$ is negative, the voltage on bus $j$ can still be maintained within the limit even if generator $i$ withholds its reactive power output. In this case, there is no voltage deficit, or $\Delta V_{i \rightarrow j}=0$. The illustrative relationships of voltage deficient $\left(\Delta V_{i \rightarrow j}\right)$, voltage $\operatorname{margin}\left(V_{j}^{0}-\underline{V_{j}}\right)$, residual voltage drop $\left(V_{i \rightarrow j}^{-}-V_{-i \rightarrow j}^{+}\right)$,

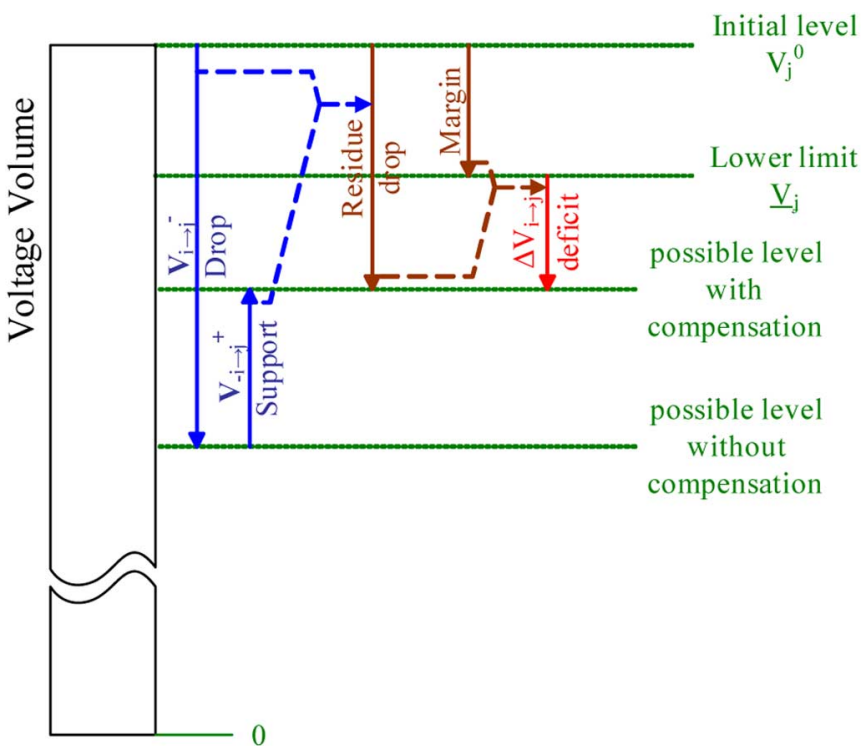

Fig. 1. Voltage relationships.

initial voltage $\left(V_{j}^{0}\right)$, voltage lower limit $\left(V_{j}\right)$, voltage drop $\left(V_{i \rightarrow j}^{-}\right)$, and voltage support $\left(V_{-i \rightarrow j}^{+}\right)$are shown in Fig. 1.

The last step is to calculate the reactive must-run capacity. If $\Delta V_{i \rightarrow j}$ is known, the corresponding amount of reactive must-run capacity (RMRC) can be obtained according to voltage sensitivity $\partial V_{j} / \partial Q_{i}$. The RMRC held by generator $i$ on node $j$ can be formulated as

$$
R M R C_{i \rightarrow j}=\frac{\Delta V_{i \rightarrow j}}{\left|\frac{\partial V_{j}}{\partial Q_{i}}\right|} .
$$

$R M R C_{i \rightarrow j}$ ranges from 0 to $Q_{i}^{0}-Q_{i}$. It is a precise index showing the magnitude of point-to-point market power, e.g., generator $i$ to bus $j$. We also notice that the initial value of voltage $V_{j}^{0}$ has very limited impacts on the values of $R M R C_{i \rightarrow j}$, i.e., the must-run capacities found using (6)-(9) are almost the same using different initial voltages. This is proved in Appendix A.

As $R M R C_{i \rightarrow j}$ does not indicate the market power of a generator, we further define $R M R C_{i}$, which can represent the reactive must-run capacity of generator $i$, as show in

$$
R M R C_{i}=\max _{j}\left(R M R C_{i \rightarrow j}, j \in \text { node }\right) .
$$

Since generator $i$ may have different values of $R M R C_{i \rightarrow j}$ on different nodes, $R M R C_{i}$ is defined as the biggest one of all $R M R C_{i \rightarrow j} . R M R C_{i}$ can be standardized by the total capacity of all generators in the system, as shown in (11), so that the reactive must-run index (RMRI) of generator $i, R M R I_{i}$ can be obtained. The value of $R M R I_{i}$ is between $0 \%$ and $100 \%$ for different generators. The higher the $R M R I_{i}$ value, the greater the proportion of must-run reactive capacity that generator $i$ holds

$$
R M R I_{i}=\frac{\max _{j}\left(R M R C_{i \rightarrow j}, j \in \text { node }\right)}{\sum_{i \in G}\left(\overline{Q_{i}}-\underline{Q_{i}}\right)}=\frac{R M R C_{i}}{\sum_{i \in G}\left(\overline{Q_{i}}-\underline{Q_{i}}\right)} .
$$


In the end, the index $R M R I_{\text {system }}$ is defined to measure the market power of the whole system. The index is defined as the ratio of the total must-run capacity over total capacity, as shown in

$$
R M R I_{\text {system }}=\frac{\sum_{i} R M R C_{i}}{\sum_{i \in G}\left(\overline{Q_{i}}-\underline{Q_{i}}\right)}=\sum_{i} R M R I_{i} .
$$

$R M R I_{\text {system }}$ is also equal to the sum of all generators' $R M R I_{i} \cdot R M R I_{\text {system }}$ is an index measuring the severity of reactive market power of an electric power system. The value of $R M R I_{\text {system }}$ is between $0 \%$ and $100 \%$. In the extreme case, $R M R I_{\text {system }}=1$ represents that all reactive power capacities in the system are must-run. $R M R I_{\text {system }}=0$ is the ideal situation that there is no must-run capacity, nor market power, in the system.

The proposed must-run indices defined in (10)-(12) can be used to study the market power held by individual generators. The indices can also be applied in a traditionally integrated power system to indicate the locations that are in shortage of reactive power sources as well as a signal for market power control and mitigation. For example, if $R M R C_{i}$ is found to be nonzero (say, 100 MVA), the system operator can mitigate the market power by entering a long term contract of 100 MVA with generator $i$.

When developing the must-run indices, we focus mainly on the reactive power capacity of generator and voltage control limits. However, some other limits, such as, line thermal limits and voltage stability limits could be more restrictive than voltage control limits. Moreover, the uncertainty of reactive power demand and the probability of system failure could also affect the reactive market power held by a generator.

In the above, RMRC index is calculated based on linearized model. Here, a complete nonlinear model is proposed for calculating RMRC index, while considering line thermal limits. For any generator, an optimization model need to be solved to obtain the minimum reactive power generation of the generator, $Q_{i} *$, hence $R M R C_{i}$. The model is shown in (13)-(18) at the bottom of the page, where subscripts $m$ and $n$ denote bus index, and $l$ denotes line index. $P_{m}^{0}$ denotes the active power scheduled for the generator at bus $m . P D_{m}$ and $Q D_{m}$ denote load at bus $m . \overline{I_{l}}$ denotes the line current limit of line $l$. BUS and LINE denote the index set of buses and lines, respectively.
By solving the nonlinear model, we can obtain the must-run reactive power of generator $i$, i.e., the minimum reactive power generation required for generator $i, Q_{i} *$. Hence, the reactive must-run capacity of generator $i$ can be obtained as

$$
R M R C_{i}= \begin{cases}Q_{i}^{*}, & Q_{i}^{*}>0 \\ 0, & Q_{i}^{*} \leq 0\end{cases}
$$

In fact, the proposed model (5)-(10) is the linear simplification of this nonlinear model (13)-(19) when phase angle differences are very small and line thermal limits are ignored. As the computation burden of the non-linear model is much bigger than the linearized model (the comparison of computation burdens and errors are given in the case studies), in the following context, we will use the linear model (5)-(10) for RMRC calculation.

\section{B. Indices for Measuring Market Power for GenCos}

In electricity markets, GenCos have their own generators. They submit generation offers on behalf of their generators with the strategy of maximizing the profit of the whole company. Among all generators owned by one GenCo, if at least one generator holds must-run VAr capacity, the GenCo obviously has reactive market power in the system operation. Moreover, a GenCo could manipulate the market even if it does not own any reactive power must-run generator. A simple example can explain this manipulation behavior. Assuming generator A and $\mathrm{B}$ are owned by same GenCo, each of them does not have must-run capacity by itself. If the GenCo withholds the reactive power output of two generators at the same time, it is possible that system voltage profiles are not able to be maintained within limits. We say that, the GenCo has market power by manipulating its generators. Different from individual must-run generators, which hold market power naturally because of their strategic locations or system load profiles, a GenCo holds market power by manipulating market or taking advantages of market structure flaws (here, market structure flaw refers to the combination of multiple generators that possibly emerge market power). Therefore, besides the RMRI proposed for individual generators, in this subsection, we will study the market power held by GenCos and propose a set of must-run indices for GenCos.

Similar as in the previous subsection, we can formulate the possible voltage drop caused by GenCo $I$ withholding reactive

$$
\begin{aligned}
\underset{Q_{m}, V_{m}, \theta_{m}}{\operatorname{Min}} & Q_{i} \\
\text { s.t. } & P_{m}^{0}-P D_{m}=V_{m} \sum_{n \in B U S} V_{n}\left(G_{m n} \cos \theta_{m n}+B_{m n} \sin \theta_{m n}\right), \quad m \in B U S \\
& Q_{m}-Q D_{m}=V_{m} \sum_{n \in B U S} V_{n}\left(G_{m n} \sin \theta_{m n}-B_{m n} \cos \theta_{m n}\right), \quad m \in B U S \\
& \left|\left(G_{m n}+j B_{m n}\right)\left[V_{m}\left(\cos \theta_{m}+j \sin \theta_{m}\right)-V_{n}\left(\cos \theta_{n}+j \sin \theta_{n}\right)\right]\right| \leq \overline{I_{l}}, \quad l \in L I N E \\
& \frac{Q_{m} \leq Q_{m} \leq \overline{Q_{m}}, m \in B U S}{}, \quad l \quad m \in B U S
\end{aligned}
$$


power, as shown in (20). Here, index set $I$ include all generators, $i \in I$, of the GenCo

$$
\begin{aligned}
V_{I \rightarrow j}^{-}=\sum_{i \in I}\left(Q_{i}^{0}-\underline{Q_{i}}\right) \cdot \max \left(\frac{\partial V_{j}}{\partial Q_{i}}, 0\right) \\
+\sum_{i \in I}\left(Q_{i}^{0}-\overline{Q_{i}}\right) \cdot \min \left(\frac{\partial V_{j}}{\partial Q_{i}}, 0\right) .
\end{aligned}
$$

In fact, equation (6) is a special case of (20) when there is only one generator $i$ owned by GenCo $I$. In most cases, a GenCo usually has more than one generator on different buses.

The voltage support from the sources other than GenCo $I$ is formulated in

$$
\begin{aligned}
V_{-I \rightarrow j}^{+}= & \sum_{k \notin I, k \in G \bigcup C}\left(\overline{Q_{k}}-Q_{k}^{0}\right) \cdot \max \left(\frac{\partial V_{j}}{\partial Q_{k}}, 0\right) \\
& +\sum_{k \notin I, k \in G \bigcup C}\left(\underline{Q_{k}}-Q_{k}^{0}\right) \cdot \min \left(\frac{\partial V_{j}}{\partial Q_{k}}, 0\right) .
\end{aligned}
$$

By summing up the negative and positive voltage deviations on $V_{j}$, the voltage deficit on bus $j$ can be obtained for the case that GenCo $I$ exercise market power, as shown in

$$
\Delta V_{I \rightarrow j}=\max \left\{0,\left(V_{I \rightarrow j}^{-}-V_{-I \rightarrow j}^{+}\right)-\left(V_{j}^{0}-\underline{V_{j}}\right)\right\} .
$$

Different from the value of $\Delta V_{i \rightarrow j}$ defined in (8), which is corresponding to the RMRC of a specific generator, a value of $\Delta V_{I \rightarrow j}$ in (22) can map to different RMRCs, depending on which generators of the GenCo are selected for RMRC calculation. Selecting different groups of generators withholding reactive power output, the values of RMRCs can be different.

For the individual generator, $R M R C_{i \rightarrow j}$ can be calculated according to the voltage sensitivity in (9), while for a GenCo, $R M R C_{I \rightarrow j}$ is calculated by finding a combination of generators that can support voltage deficit with the minimal reactive power capacity, i.e., $R M R C_{I \rightarrow j}$.

If all voltage sensitivities are positive, the minimal must-run capacity that GenCo $I$ held on node $j$ can be obtained by an optimization model described as

$$
\begin{array}{ll}
\text { Min } & \operatorname{RMRC}_{I \rightarrow j}=\sum_{i \in \Omega} Q_{i}-\underline{Q_{i}} \\
\text { s.t. } & \sum_{i \in \Omega}\left(Q_{i}-\underline{Q_{i}}\right) \cdot \max \left(\frac{\partial V_{j}}{\partial Q_{i}}, 0\right) \geq \Delta V_{I \rightarrow j} \\
& \Omega=\left\{\frac{i \mid \partial V_{j}}{\partial Q_{i}}>0, i \in I\right\} .
\end{array}
$$

The objective is to obtain the must-run reactive power capacity of GenCo $I$, so that the voltage support of GenCo $I$ on bus $j$ is higher than the voltage deficit of bus $j$, i.e., constraint is satisfied.

Considering both positive voltage sensitivities and negative voltage sensitivities, the optimization model (23)-(24) can be modified as

$$
\text { Min } \quad R M R C_{I \rightarrow j}=\sum_{m \in \Omega}\left(Q_{m}-\underline{Q_{m}}\right)+\sum_{n \in \Lambda}\left(\overline{Q_{n}}-Q_{n}\right)
$$

$$
\begin{array}{ll}
\text { s.t. } \quad & \sum_{m \in \Omega}\left(Q_{m}-\underline{Q_{m}}\right) \cdot \max \left(\frac{\partial V_{j}}{\partial Q_{m}}, 0\right) \\
& +\sum_{n \in \Lambda}\left(Q_{n}-\underline{Q_{n}}\right) \cdot \min \left(\frac{\partial V_{j}}{\partial Q_{n}}, 0\right) \geq \Delta V_{I \rightarrow j} \\
\Omega & =\left\{m \mid \frac{\partial V_{j}}{\partial Q_{m}}>0, m \in I\right\} \\
\Lambda & =\left\{n \mid \frac{\partial V_{j}}{\partial Q_{n}}>0, n \in I\right\} .
\end{array}
$$

Optimization model (25)-(26) is a linear programming (LP) problem. By solving the LP problem, the optimal combination of generators and their optimal generations $Q_{i}^{*}(i \in I)$ are obtained. The minimal reactive must-run capacity held by GenCo $I$ on node $j$ can therefore be formulated as

$$
R M R C_{I \rightarrow j}=\sum_{m \in \Omega}\left(Q_{m}^{*}-\underline{Q_{m}}\right)+\sum_{n \in \Lambda}\left(\overline{Q_{n}}-Q_{m}^{*}\right) .
$$

Similarly, we propose three indices: reactive must-run capacity for GenCo $I\left(R M R C_{I}\right)$, reactive must-run index for GenCo $I\left(R M R I_{I}\right)$, and reactive must-run index for the system $\left(R M R I_{\text {system }}\right)$. They are formulated as

$$
\begin{aligned}
R M R C_{I} & =\max _{j}\left(R M R C_{I \rightarrow j}, j \in \text { node }\right) \\
R M R I_{I} & =\frac{\max _{j}\left(R M R C_{I \rightarrow j}, j \in \text { node }\right)}{\sum_{i \in G}\left(\overline{Q_{i}}-\underline{Q_{i}}\right)} \\
& =\frac{R M R C_{I}}{\sum_{i \in G}\left(\overline{Q_{i}}-\underline{Q_{i}}\right)} \\
R M R I_{\text {system }} & =\frac{\sum_{I} R M R C_{I}}{\sum_{i \in G}\left(\overline{Q_{i}}-\underline{Q_{i}}\right)}=\sum_{I} R M R I_{I} .
\end{aligned}
$$

In addition to withholding reactive power output, a GenCo can also exercise market power by "not connecting" some of its generators (for example, by not submitting offers or by strategic unit maintenances), so that some other generators owned by it become must-run units. Similar to (20), the possible voltage deficit of this case is formulated in

$$
\begin{aligned}
V_{I \rightarrow j}^{-}= & \sum_{p \in \Phi}\left(Q_{p}^{0}-\underline{Q_{p}}\right) \cdot \max \left(\frac{\partial V_{j}}{\partial Q_{p}}, 0\right) \\
& +\sum_{p \in \Phi}\left(Q_{p}^{0}-\overline{Q_{p}}\right) \cdot \min \left(\frac{\partial V_{j}}{\partial Q_{p}}, 0\right) \\
& +\sum_{q \in \Delta} Q_{q}^{0} \cdot \max \left(\frac{\partial V_{j}}{\partial Q_{q}}, 0\right) \\
& +\sum_{q \in \Delta} Q_{q}^{0} \cdot \min \left(\frac{\partial V_{j}}{\partial Q_{q}}, 0\right)
\end{aligned}
$$

where $\Phi$ and $\Delta$ are the subsets of $I . \Phi$ is the index set of the generators that are connected to the system, and $\Delta$ includes the other generators that are "disconnected" for reactive power withholding purpose. $\Phi \cup \Delta=I$, and $\Phi \cap \Delta=0$. The voltage deficit $V_{I \rightarrow j}^{-}$in (31) can be greater than that in (20) if $Q_{q}>0$ for positive $\partial V_{j} / \partial Q_{q}$ and $\overline{Q_{q}}<0$ for negative $\partial V_{j} / \partial \overline{Q_{q}}$. 
In the case that a GenCo exercise market power by not connecting, model (25)-(26) is modified and described as

$$
\begin{aligned}
\text { Min } & R M R C_{I \rightarrow j}=\sum_{m \in \Omega^{\prime}}\left(Q_{m}-\underline{Q_{m}}\right)+\sum_{n \in \Lambda^{\prime}}\left(\overline{Q_{n}}-Q_{n}\right) \\
\text { s.t. } & \sum_{m \in \Omega^{\prime}}\left(Q_{m}-\underline{Q_{m}}\right) \cdot \max \left(\frac{\partial V_{j}}{\partial Q_{m}}, 0\right) \\
& +\sum_{n \in \Lambda^{\prime}}\left(Q_{n}-\overline{Q_{n}}\right) \cdot \min \left(\frac{\partial V_{j}}{\partial Q_{n}}, 0\right) \geq \Delta V_{I \rightarrow j} \\
\Omega^{\prime} & =\left\{m \mid \frac{\partial V_{j}}{\partial Q_{m}}>0, m \in \Phi\right\} \\
\Lambda^{\prime} & =\left\{n \mid \frac{\partial V_{j}}{\partial Q_{n}}<0, n \in \Phi\right\} .
\end{aligned}
$$

Since $\Phi \subset I$, the optimal $R M R C_{\Phi \rightarrow j}$ value of (32) might be bigger than that of (25) (even if $V_{I \rightarrow j}^{-}$is the same) in the condition that generators with bigger $\partial V_{j} / \partial Q_{m}$ are "not connected," i.e., these generators are not in the subset $\Phi$. In this way, by disconnecting generators, a GenCo can increase its market power in the system, or even, a GenCo originally without market power can create market power.

In the IEEE 118-bus system-based capability reactive power market proposed by Ahmed and Strbac [3], two of the five generators owned by a generation company were found having market power. When the capability price offered by the two generators increase from 0.1 to 100 p.u., they are always being selected due to their strategic locations in providing reactive power. The following is mentioned in the paper.

It is important to mention that these two generators, irrespective of their high bid price, remain to be required for the provision of reactive reserve only as a part of the group.

This is a case of market power held by a GenCo. The proposed indices will be used to test the market power held by the GenCo in the IEEE 118-bus system in case studies.

\section{CASE STUdies}

In this section, we will apply the proposed reactive power must-run indices to the Nordic 32-bus system and the IEEE 118-bus system to test the market power held by individual generators and GenCos, respectively.

\section{A. Reactive Market Power in the Nordic 32-Bus System}

In the Nordic system shown in Fig. 2, there are twenty generators. We assume that each generator is an independent generation company. The market power in the system is caused by the generator strategic bidding. In [5] and [7], by analyzing the uniform reactive market prices, the authors have found that some generators in the Nordic system hold reactive market power. They can raise market prices by offering higher prices. In this numerical example, we will study the market power of the system using the proposed indices and compare the results with that obtained in [5].

Based on the proposed index formulas (9)-(12), the reactive must-run indices $R M R C_{i \rightarrow j}, R M R I_{i}, R M R I_{\text {system }}$, and

\begin{tabular}{|c|c|c|c|c|}
\hline \multicolumn{4}{|c|}{$R M R C_{i \rightarrow j}$ (MVAr) } & \multirow{2}{*}{$R M R I_{i}$} \\
\hline Gen $i$ & 1042 & 2032 & 4072 & \\
\hline 1042 & 14.436 & 0 & 0 & 0.001361 \\
\hline 2032 & 0 & 28.334 & 0 & 0.002671 \\
\hline 4072 & 0 & 0 & 87.285 & 0.008227 \\
\hline$\frac{\sum_{i \in G} \Delta V_{i \rightarrow j}}{V_{j}^{0}}$ & 0.014207 & 0.016769 & 0.014026 & $\begin{array}{l}R M R I_{\text {system }} \\
=0.0123\end{array}$ \\
\hline
\end{tabular}

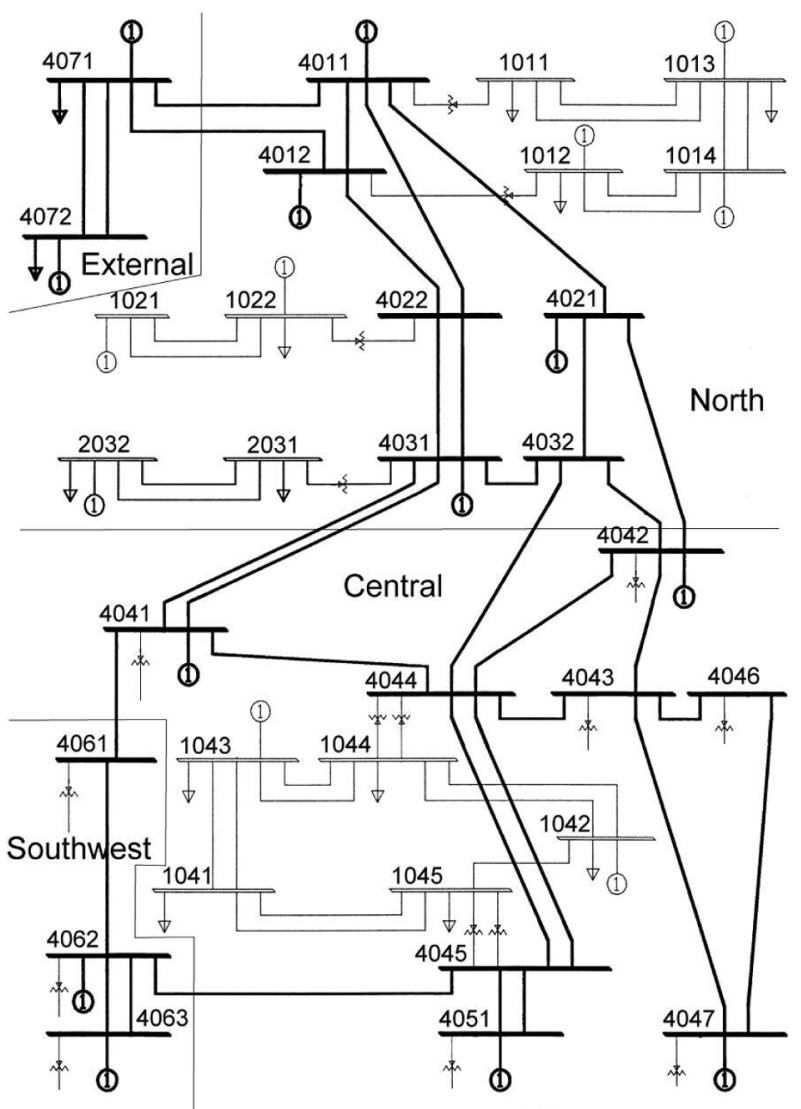

Fig. 2. Nordic 32-bus system.

TABLE I

Must-Run INDices for THE BusEs HAVING MARKET POWER

$\sum_{i \in G} \Delta V_{i \rightarrow j} / V_{j}^{0}$ are calculated for the Nordic system. The results are given in Table I. Since only generators on nodes 1042, 2032, and 4072 are found having market power holding reactive power, the results of other nodes, which are all zeros, are not listed in the table.

From Table I, we can find the following.

1) The non-zero values of indices $R M R I_{i}$ for Gen 4072 , Gen2032 and Gen1041 show that the three generators have market power in the system. This result is exactly the same as the results obtained in [5] and [7], in which the market power of Nordic system was studied according to generators' abilities of manipulating market prices.

2) Each non-zero $R M R C_{i \rightarrow j}$ turns out at $i=j$, which shows that reactive market power has local nature.

3) The generator at bus 4072 holds the largest reactive must-run capacity (87.285 MVAr), but it does not mean 
that bus 4072 suffers the most severe voltage problem. We have noticed that

$$
\frac{\sum_{i \in G} \Delta V_{i \rightarrow 2032}}{V_{2032}^{0}}>\frac{\sum_{i \in G} \Delta V_{i \rightarrow 1042}}{V_{1042}^{0}}>\frac{\sum_{i \in G} \Delta V_{i \rightarrow 4072}}{V_{4072}^{0}} .
$$

The value of $\sum \Delta V_{i \rightarrow j} / V_{j}^{0}$ show that bus 2032 has the most severe voltage problem. In practical system operation, the generator at bus 2032 is operated as a synchronous condenser for reactive power compensation. The highest $R M R I_{i}$ value of Gen4072 is due to its largest generation capacity in the system. This is also the reason that we propose not only $R M R I_{i}$ index but also $\sum \Delta V_{i \rightarrow j} / V_{j}^{0}$ index to measure market power from both capacity and voltage view points.

It has been found in [5] and [7] that the three generators found in above (especially Gen4072) can always be price-makers even if they offer very high prices. With the market analysis method used in [5] and [7], to find the market power holders, the system operator needs to simulate the market model for many times with various offer price combinations of all generators.

The proposed must-run indices have the advantages of 1) requiring less computation effort in measuring reactive market power; 2) finding market power holders without running market simulation. Since the proposed market power measurement method is mainly based on the network structure, the results are independent of either reactive power settlement mechanisms or the price offers of generators.

1) Market Power in Different Reactive Power Procurement Models: The criterion of being a good market power measurement method is that the results obtained by the method are independent of the way of procuring reactive power. Considering currently there is no uniform reactive power payment mechanism or reactive power market, we test the results of the proposed must-run indices under different reactive power procurement mechanisms.

The RMRIs are calculated for three reactive power procurement models: Model A, Model B and Model C. The results of $R M R I_{4072}, R M R I_{2032}, R M R I_{1042}$, and $R M R I_{\text {system }}$ for the models are shown in Fig. 3.

Model A: Min $\sum Q_{G, i}$, minimize total reactive power procurement of generators.

Model B: Min $\sum\left(Q_{G, i}+Q_{C, i}\right)$, minimize total reactive power procurement of generators and capacitors.

Model C: Min $\sum P_{\text {loss }}$, minimize system losses.

The optimization results of the three models, e.g., voltages, phase angles, real power outputs, and reactive power outputs of the generators are significantly different. However, as shown in Fig. 3, the values of RMRIs are very similar using three different reactive power procurement methods. The results show that the proposed must-run indices are independent of reactive power procurement and system operation. The dominant factors that make one generator "must-run" are the location of the generator and the system demand.

In Fig. 3, there are small differences between the indices obtained from different models. We believe that it is because of the linearization approximation of voltage sensitivity $\partial V_{j} / \partial Q_{i}$. To find out how big the deviation is caused by the linearization approximation, we have calculated the RMRI indices using

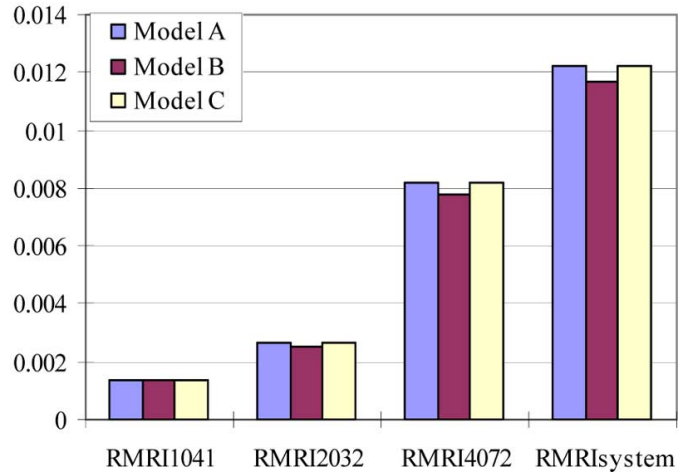

Fig. 3. Must-run indices for different operation models.

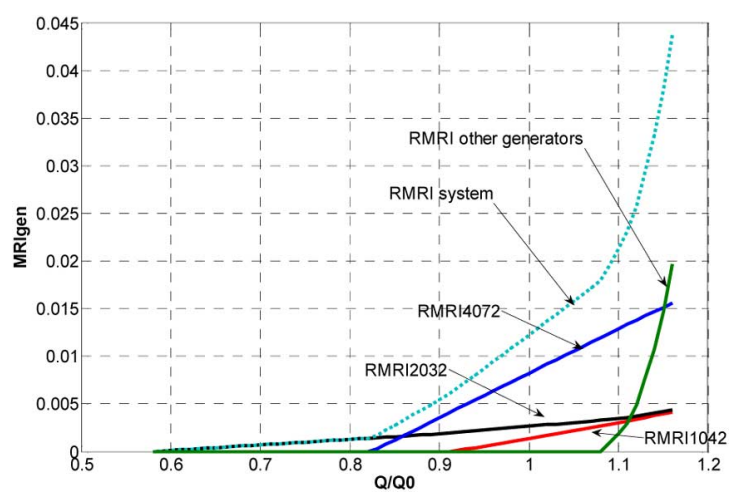

Fig. 4. Must-run indices with reactive power demand changes.

both linear model (9)-(12) and the nonlinear model (13)-(19). It is found that the errors of index values caused by linearization are about $2.313 \%$ to $7.157 \%$. However, the computation time of using nonlinear model is much higher (about 62 to 304 times) than using linearized approach. Considering the time consumption, the $2 \%-7 \%$ of deviation of linearization is acceptable, and it is good enough for the purpose of indicating who holds market power and its level.

2) Impacts of Demand Variations on Market Power: In a power system, reactive power demand may vary from time to time. At different demand levels, market power holders may be different, and their abilities of manipulating the market may also be different. In this example, we will test how reactive must-run indices change if the reactive power demand on each bus is increased gradually from 0 to $1.2 * Q_{0}$, where $Q_{0}$ is the original demand. The simulation results are shown in Fig. 4, which shows how $R M R I_{4072}, R M R I_{2032}, R M R I_{1042}$, and $R M R I_{\text {system }}$ change when the per unit reactive demand, $Q / Q_{0}$ increases.

Fig. 4 shows that reactive power demand has significant impacts on RMRI indices. The following are found.

1) When reactive power demand is lower than $0.58 * Q_{0}$, there is no must-run generator in the system.

2) From the demand level $0.58 * Q_{0}$, the first must-run generator, Gen2032 starts to show its market power. The highest value of $\sum \Delta V_{i \rightarrow j} / V_{j}^{0}$ of Gen2032 in Table I also show that the generator has strong ability to affect voltages. In the real Nordic system, Gen2032 is in fact operated as a synchronous condenser for providing reactive power. 


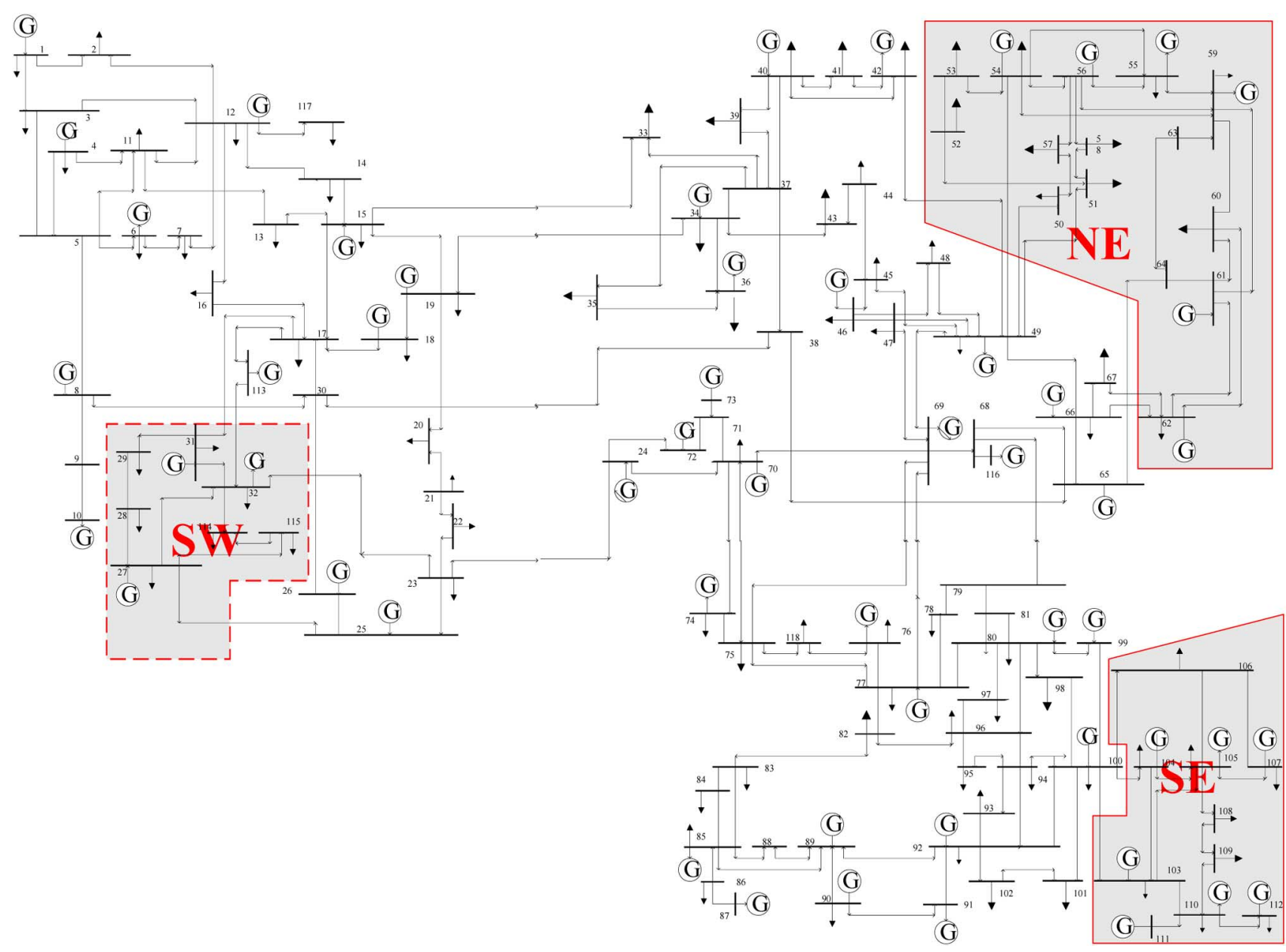

Fig. 5. IEEE 118-bus system.

3) When the demand reaches $0.83 * Q_{0}$, Gen 4072 starts to become must-run generator; then, Gen1042 at $0.92 * Q_{0}$.

4) When the demand is higher than $1.08 * Q_{0}$, more generators become must-run generators resulting in the dramatically increase of system index $R M R I_{\text {system }}$, as the dotted line shown in Fig. 4,

5) There is no feasible solution to maintain the system voltages within $(0.95,1.05)$ when reactive power demand is higher than $1.17 * Q_{0}$.

\section{B. Market Power Analysis for the IEEE 118-Bus System}

In this subsection, we will use the IEEE 118-bus system (as shown in Fig. 5) to analyze the market power held by a GenCo owning multiple generators at different buses.

The IEEE 118-bus system is an ideal system that has adequate reactive power capacities for normal operations. However, when calculating the initial values, we have noticed that some areas (the buses with close electrical distances) usually only have net injections of reactive power. The buses in these areas have relatively low voltages and weak connections to other buses. These areas are highlighted with gray shade and named as NE, SE and SW in Fig. 5. We will test if market power exists if some of the generators in the areas are owned by same GenCo and they manipulate reactive power outputs simultaneously.
In the normal operating states, there is no reactive must-run generator due to sufficient reactive power supply in the system. The reactive must-run indices obtained using (9)-(12) for the system have proved this. In [3], the reactive market power was tested for the IEEE 118-bus system under ten contingency states; six generators (Gen54, Gen59, Gen61, Gen55, Gen56 and Gen62) were assumed to be owned by one GenCo and they increased reactive power price offers simultaneously. It was found in [3] that when the offer prices were increased to a certain level, further increase of the price did not change the clearing amount sold by this GenCo. The contingencies used in this paper maybe different from that used in [3]. The details of the ten contingencies, e.g., which facilities are outage, are not provided in [3].

In this paper, we first assume that a GenCo in NE Area owns three generators, Gen54, Gen59 and Gen61. Using (28)-(30), the reactive must-run indices are calculated for the GenCo under three contingency states, line 49-50 outage, line 49-51 outage, and line 49-54 outage. The must-run indices are given in Table II. The values of RMRC and RMRI show that the GenCo has market power. This result is in accord with the simulation results in [3].

Then, we test the situation if the three generators are independent, in other words, they are not owned by any GenCo. It is found that there is no must-run capacity for each generator under 
TABLE II

REACTIVE MUST-RUN INDICES FOR THE IEEE 118-BUS SYSTEM

\begin{tabular}{|c|c|c|c|c|}
\hline Area & $\begin{array}{c}\text { Contingency } \\
\text { outage }\end{array}$ & $\begin{array}{c}\text { Generators possessed by } \\
\text { one GenCo in the Area }\end{array}$ & $\begin{array}{c}\text { RMRC } \\
(\text { MVAr) }\end{array}$ & RMRI(\%) \\
\hline NE & $49-50$ & {$[54,59,61]$} & 4.27 & 0.22 \\
\hline NE & $49-51$ & {$[54,59,61]$} & 29.48 & 1.54 \\
\hline NE & $49-54$ & {$[54,59,61]$} & 3.2 & 0.17 \\
\hline NE & No outage & {$[54,59,61]$} & 0 & 0 \\
\hline SW & $17-31 \& 25-27$ & {$[27,31]$} & 22.65 & 1.18 \\
\hline SE & Gen100 & {$[112,111,107,103]$} & 2.96 & 0.15 \\
\hline SE & Gen100 & {$[112,111,107,103,104]$} & 16.00 & 0.84 \\
\hline SE & Gen100 & {$[112,111,107,103,104,105]$} & 35.42 & 1.85 \\
\hline
\end{tabular}

the same contingencies. This shows that a GenCo having generators on multiple nodes could exercise reactive market power, although the generators themselves are not reactive must run.

Furthermore, the market power held by the GenCo is tested for area SW and area SE. The results are given in Table II. In area SE, assume an outage of Gen 100 , the reactive must-run indices are calculated and compared if different numbers of generators are owned by the GenCo. In the case that the GenCo owns four generators, Gen112, Gen111, Gen107 and Gen103, the must-run capacity of the GenCo is 2.96MVAr. If the GenCo owns one more generator, Gen104, the must-run capacity increases to 16MVAr. And if it owns another more generator, Gen105, the must-run capacity increases to 35.42MVAr.

Based on the studies on the IEEE 118-bus system, the following is found.

- For a system with sufficient reactive power supply, although there is no natural reactive market power, it is possible for a GenCo to manipulate reactive power market and hold must-run capacity by controlling its generators located at different buses.

- Even if such a GenCo has no market power in normal operating state, it may have market power in some critical contingency states, as shown in the tests for area NE in Table II.

- The market power held by a GenCo increases if the numbers or the sizes of the generators it owns increase.

\section{Discussions}

The Nordic 32-bus system and the 118-bus system have been used to test the proposed reactive must-run indices. It can be found that the reactive power sources at certain locations may hold reactive market power due to the local nature of reactive power supply. The heavier the demand in the system, the more the market power holders.

For a system with sufficient reactive power capacity and no market power in the normal state, contingency conditions may make some generators held must-run capacity, or worsen the existing market power problem.

Furthermore, market structure can cause market power even in a topologically ideal system. The numerical examples of the IEEE 118-bus system show that, a GenCo with a big market share and at certain locations can manipulate the reactive power to a certain extent. The system will be vulnerable to market power abuse if a single GenCo holds a big share of reactive power capacities in an area with relatively weak reactive power support.

\section{CONCLUSIOnS}

In this paper, the must-run concept is applied to analyze reactive market power in power system operation. The reactive power must-run indices are proposed for individual generators and GenCos, respectively. It is shown that the values of reactive power must-run indices keep unchanged when reactive power outputs and voltages change. A GenCo having generators at different nodes can exercise market power by controlling the outputs of its generators.

The case studies analyzed the reactive market power caused by system structure deficiency and market structure. The results are consistent with the real system operations and the results in the references, in which market power are measured using other methods.

\section{APPENDIX}

Relationship of RMRC Index and Initial Voltages: In this subsection, we will prove that different initial voltages $V_{j}^{0}$ have very limited impacts on the value of $R M R C_{i \rightarrow j}$ index proposed by (6)-(9).

Assume there are two sets of different initial points $\left(V^{A}, Q^{A}\right)$ and $\left(V^{B}, Q^{B}\right)$. For generator $i$ and bus $j$, the values of $R M R C_{i \rightarrow j}$ can be calculated based on two initial points $\mathrm{A}$ and $\mathrm{B}$, respectively

$$
\begin{aligned}
\operatorname{RMRC}_{i \rightarrow j}^{A} & =\frac{\Delta V_{i \rightarrow j}^{A}}{\left|\frac{\partial V_{j}}{\partial Q_{i}}\right|} \\
\operatorname{RMRC}_{i \rightarrow j}^{B} & =\frac{\Delta V_{i \rightarrow j}^{B}}{\left|\frac{\partial V_{j}}{\partial Q_{i}}\right|} .
\end{aligned}
$$

To calculate the difference of the RMRC values between two initial points, subtracting (34) by (35), we obtain

$$
R M R C_{i \rightarrow j}^{A}-R M R C_{i \rightarrow j}^{B}=\frac{\Delta V_{i \rightarrow j}^{A}-\Delta V_{i \rightarrow j}^{B}}{\left|\frac{\partial V_{j}}{\partial Q_{i}}\right|} .
$$

Based on (8), the numerator of (36) can be formulated as

$$
\begin{aligned}
& \Delta V_{i \rightarrow j}^{A}-\Delta V_{i \rightarrow j}^{B} \\
& \quad=\max \left\{0,\left(V_{i \rightarrow j}^{-A}-V_{-i \rightarrow j}^{+A}\right)-\left(V_{j}^{A}-\underline{V_{j}}\right)\right\} \\
& \quad-\max \left\{0,\left(V_{i \rightarrow j}^{-B}-V_{-i \rightarrow j}^{+B}\right)-\left(V_{j}^{B}-\underline{V_{j}}\right)\right\} .
\end{aligned}
$$

The non-zero terms of (37) can be organized as

$$
\begin{aligned}
\left(V_{i \rightarrow j}^{-A}-V_{-i \rightarrow j}^{+A} V_{j}^{A}+\underline{V_{j}}\right) \\
-\left(V_{i \rightarrow j}^{-B}-V_{-i \rightarrow j}^{+B}-V_{j}^{B}+\underline{V_{j}}\right) \\
\quad=\left(V_{i \rightarrow j}^{-A}-V_{i \rightarrow j}^{-B}\right)-\left(V_{-i \rightarrow j}^{+A}-V_{-i \rightarrow j}^{+B}\right) \\
\quad-\left(V_{j}^{A}-V_{j}^{B}\right) .
\end{aligned}
$$


There are three terms on the right side of the equation (38). The first term can be expanded as (39) according to (6) and the second term can be expanded as (40) according to (7)

$$
\begin{aligned}
& \left(V_{i \rightarrow j}^{-A}-V_{i \rightarrow j}^{-B}\right) \\
& =\left(Q_{i}^{A}-\underline{Q_{i}}\right) \cdot \max \left(\frac{\partial V_{j}}{\partial Q_{i}}, 0\right) \\
& +\left(Q_{i}^{A}-\overline{Q_{i}}\right) \cdot \min \left(\frac{\partial V_{j}}{\partial Q_{i}}, 0\right) \\
& -\left(Q_{i}^{B}-\underline{Q_{i}}\right) \cdot \max \left(\frac{\partial V_{j}}{\partial Q_{i}}, 0\right) \\
& -\left(Q_{i}^{B}-\overline{Q_{i}}\right) \cdot \min \left(\frac{\partial V_{j}}{\partial Q_{i}}, 0\right) \\
& =\left(Q_{i}^{A}-Q_{i}^{B}\right) \cdot \max \left(\frac{\partial V_{j}}{\partial Q_{i}}, 0\right) \\
& +\left(Q_{i}^{A}-Q_{i}^{B}\right) \cdot \min \left(\frac{\partial V_{j}}{\partial Q_{i}}, 0\right) \\
& =\left(Q_{i}^{A}-Q_{i}^{B}\right) \cdot \frac{\partial V_{j}}{\partial Q_{i}} \\
& \left(V_{-i \rightarrow j}^{+A}-V_{-i \rightarrow j}^{+B}\right) \\
& =\sum_{k \neq i, k \in G \bigcup C}\left(\overline{Q_{k}}-Q_{k}^{A}\right) \cdot \max \left(\frac{\partial V_{j}}{\partial Q_{k}}, 0\right) \\
& +\sum_{k \neq i, k \in G \bigcup C}\left(\underline{Q_{k}}-Q_{k}^{A}\right) \cdot \min \left(\frac{\partial V_{j}}{\partial Q_{k}}, 0\right) \\
& \text { - } \sum_{k \neq i, k \in G \bigcup C}\left(\overline{Q_{k}}-Q_{k}^{B}\right) \cdot \max \left(\frac{\partial V_{j}}{\partial Q_{k}}, 0\right) \\
& \text { - } \sum_{k \neq i, k \in G \bigcup C}\left(\underline{Q_{k}}-Q_{k}^{B}\right) \cdot \min \left(\frac{\partial V_{j}}{\partial Q_{k}}, 0\right) \\
& =\sum_{k \neq i, k \in G \bigcup C}\left(Q_{k}^{B}-Q_{k}^{A}\right) \cdot \max \left(\frac{\partial V_{j}}{\partial Q_{k}}, 0\right) \\
& +\sum_{k \neq i, k \in G \bigcup C}\left(Q_{k}^{B}-Q_{k}^{A}\right) \cdot \min \left(\frac{\partial V_{j}}{\partial Q_{k}}, 0\right) \\
& =\sum_{k \neq i, k \in G \bigcup C}\left(Q_{k}^{B}-Q_{k}^{A}\right) \cdot \frac{\partial V_{j}}{\partial Q_{k}} \text {. }
\end{aligned}
$$

Subtracting (39) by (40), we have

$$
\begin{aligned}
& \left(V_{i \rightarrow j}^{-A}-V_{i \rightarrow j}^{-B}\right)-\left(V_{-i \rightarrow j}^{+A}-V_{-i \rightarrow j}^{+B}\right) \\
& \quad=\left(Q_{i}^{A}-Q_{i}^{B}\right) \cdot \frac{\partial V_{j}}{\partial Q_{i}}-\sum_{k \neq i, k \in G \cup C}\left(Q_{k}^{B}-Q_{k}^{A}\right) \cdot \frac{\partial V_{j}}{\partial Q_{k}} \\
& \quad=\sum_{k \in G \cup C}\left(Q_{k}^{A}-Q_{k}^{B}\right) \cdot \frac{\partial V_{j}}{\partial Q_{k}} .
\end{aligned}
$$

Using a vector to represent the result of (41)

$$
\begin{aligned}
& \left(V_{i \rightarrow j}^{-A}-V_{i \rightarrow j}^{-B}\right)-\left(V_{-i \rightarrow j}^{+A}-V_{-i \rightarrow j}^{+B}\right) \\
& \quad=\sum_{k \in G \cup C}\left(Q_{k}^{A}-Q_{k}^{B}\right) \cdot\left(\frac{\partial V_{j}}{\partial Q_{k}}\right) \\
& \quad=\left[\begin{array}{lllll}
\frac{\partial V_{j}}{\partial Q_{1}} & \cdots & \frac{\partial V_{j}}{\partial Q_{k}} & \cdots & \frac{\partial V_{j}}{\partial Q_{K}}
\end{array}\right]\left[\begin{array}{c}
Q_{1}^{A}-Q_{1}^{B} \\
\vdots \\
Q_{k}^{A}-Q_{k}^{B} \\
\vdots \\
Q_{K}^{A}-Q_{K}^{B}
\end{array}\right]
\end{aligned}
$$

Based on the Jacobian matrix in the simplified decoupled Newton-Raphson algorithm for load flow calculation [14] and the approximation of the decoupled sub-matrix of Jacobian matrix $\mathbf{J}_{22}$, equation (43) can be obtained as follows:

$$
\left(V_{i \rightarrow j}^{-A}-V_{i \rightarrow j}^{-B}\right)-\left(V_{-i \rightarrow j}^{+A}-V_{-i \rightarrow j}^{+B}\right) \approx V_{j}^{A}-V_{j}^{B} .
$$

Substituting (43) in (38), we can obtain

$$
\begin{aligned}
\left(V_{i \rightarrow j}^{-A}-V_{-i \rightarrow j}^{+A}-\right. & \left.V_{j}^{A}+\underline{V_{j}}\right) \\
& -\left(V_{i \rightarrow j}^{-B}-V_{-i \rightarrow j}^{+B}-V_{j}^{B}+\underline{V_{j}}\right) \approx 0 .
\end{aligned}
$$

Equation (44) is equivalent to

$$
\begin{aligned}
\left(V_{i \rightarrow j}^{-A}-V_{-i \rightarrow j}^{+A}\right)- & \left(V_{j}^{A}-\underline{V_{j}}\right) \\
& \approx\left(V_{i \rightarrow j}^{-B}-V_{-i \rightarrow j}^{+B}\right)-\left(V_{j}^{B}-\underline{V_{j}}\right) .
\end{aligned}
$$

From (45) and (37), we have

$$
\Delta V_{i \rightarrow j}^{A}-\Delta V_{i \rightarrow j}^{B} \approx 0 .
$$

Replace (46) in (36), we can find that the difference of the proposed must-run indices with two different initial operation points is approximately equal to zero, as shown in

$$
\begin{aligned}
R M R C_{i \rightarrow j}^{A}-R M R C_{i \rightarrow j}^{B} & =\frac{\Delta V_{i \rightarrow j}^{A}-\Delta V_{i \rightarrow j}^{B}}{\left|\frac{\partial V_{j}}{\partial Q_{i}}\right|} \approx 0 \\
& \Rightarrow R M R C_{i \rightarrow j}^{A} \approx R M R C_{i \rightarrow j}^{B} .
\end{aligned}
$$

So that the must-run capacity of a generator is not much related to the initial operating point. Using $\mathbf{B}$ matrix for the approximation is acceptable for market power calculation, which analyzes the static state of the system.

\section{REFERENCES}

[1] NERC Operating Policy-10 on Interconnected Operation Services, North American Electrical Reliability Council, 2000, Draft-3.1.

[2] National Electricity Market Ancillary Services, National Electricity Market Management Co., , Australia, 1999, Tech. Rep.

[3] S. Ahmed and G. Strbac, "A method for simulation and analysis of reactive power market," IEEE Trans. Power Syst., vol. 15, no. 3, pp. 1047-1052, Aug. 2000.

[4] J. B. Gil, T. G. S. Roman, J. J. A. Rios, and P. S. Martin, "Reactive power pricing: a conceptual framework for remuneration and charging procedures," IEEE Trans. Power Syst., vol. 15, no. 2, pp. 483-489, May 2000

[5] J. Zhong and K. Bhattacharya, "Toward a competitive market for reactive power," IEEE Trans. Power Syst., vol. 17, no. 4, pp. 1206-1215, Nov. 2002 
[6] K. Bhattacharya and J. Zhong, "Reactive power as an ancillary service," IEEE Trans. Power Syst., vol. 16, no. 2, pp. 294-300, May 2001.

[7] J. Zhong, E. Nobile, A. Bose, and K. Bhattacharya, "Localized reactive power markets using the concept of voltage control areas," IEEE Trans. Power Syst., vol. 19, no. 3, pp. 1555-1561, Aug. 2004.

[8] S. Stoft, Power System Economics. New York: Wiley, 2002.

[9] R. S. Pindyck, D. L. Rubinfeld, and W. T. H. Koh, Microeconomics. Englewood Cliffs, NJ: Prentice-Hall, 2006.

[10] D. Gan and D. V. Bourcier, "Locational market power screening and congestion management: Experience and suggestions," IEEE Trans. Power Syst., vol. 17, no. 1, pp. 180-185, Feb. 2002.

[11] P. Wang, Y. Xiao, and Y. Ding, "Nodal market power assessment in electricity markets," IEEE Trans. Power Syst., vol. 19, no. 3, pp. 1373-1379, Aug. 2004.

[12] F. L. A. T. Overbye, "Measuring reactive market power," in Proc. IEEE Power Eng. Soc. Winter Meeting, 1999, vol. 1, pp. 294-296.

[13] G. Chicco, "A new method for reactive market power assessment in competitive electricity markets," in Proc. 12th IEEE Mediterranean Electrotechnical Conf., May 12-15, 2004, vol. 3, pp. 1061-1064.

[14] A. R. Bergen and V. Vittal, Power Systems Analysis, 2nd ed. Englewood Cliffs, NJ: Prentice-Hall, 2000.

Donghan Feng received the B.Sc. degree in the Department of Electrical Engineering from Zhejiang University, Hangzhou, China, in 2003. He is currently pursuing the Ph.D. degree at Zhejiang University.

During 2006 to 2007, he worked at University of Hong Kong as a visiting scholar. His research interests are engineering and economic issues in electricity markets.
Jin Zhong (S'00-M'04) received the B.Sc. degree from Tsinghua University, Beijing, China, in 1995, the M.Sc. degree from China Electric Power Research Institute, Beijing, in 1998, and the Ph.D degree from Chalmers University of Technology, Gothenburg, Sweden, in 2003.

At present, she is an Assistant Professor in the Department of Electrical and Electronic Engineering of the University of Hong Kong. Her areas of interest are electricity sector deregulation, ancillary service pricing, and power system planning.

Deqiang Gan received the Ph.D. degree in electrical engineering from Xian Jiaotong University, Xian, China, in 1994.

He has been with the faculty of Zhejiang University, Hangzhou, China, since 2002. He visited the University of Hong Kong in 2004. He was a Senior Analyst in ISO New England, Inc., from 1998 to 2002. He held research positions in Ibraki University, University of Central Florida, and Cornell University from 1994 to 1998. His research interests are in the applications of nonlinear system and game theory to power systems.

Dr. Gan is currently on the editorial board of the European Transactions on Electric Power. 